\title{
Video Article \\ Induction of Complete Transection-Type Spinal Cord Injury in Mice
}

\author{
Ronak Reshamwala ${ }^{1,2,3}$, Tanja Eindorf ${ }^{2,3}$, Megha Shah ${ }^{2,3}$, Graham Smyth ${ }^{2,3}$, Todd Shelper ${ }^{2,3}$, James St. John*1,2,3, Jenny Ekberg ${ }^{\star 2,3}$ \\ ${ }^{1}$ Griffith Institute for Drug Discovery, Griffith University \\ ${ }^{2}$ Menzies Health Institute Queensland, Griffith University \\ ${ }^{3}$ Clem Jones Centre for Neurobiology and Stem Cell Research, Griffith University \\ * These authors contributed equally
}

Correspondence to: James St. John at j.stjohn@griffith.edu.au

URL: https://www.jove.com/video/61131

DOI: doi:10.3791/61131

Keywords: Neuroscience, Issue 159, Transverse injury, protocol, laminectomy, surgery, C57BL/6 mice, neurotrauma

Date Published: 5/6/2020

Citation: Reshamwala, R., Eindorf, T., Shah, M., Smyth, G., Shelper, T., St. John, J., Ekberg, J. Induction of Complete Transection-Type Spinal Cord Injury in Mice. J. Vis. Exp. (159), e61131, doi:10.3791/61131 (2020).

\section{Abstract}

Spinal cord injury $(\mathrm{SCl})$ largely leads to irreversible and permanent loss of function, most commonly as a result of trauma. Several treatment options, such as cell transplantation methods, are being researched to overcome the debilitating disabilities arising from SCl. Most preclinical animal trials are conducted in rodent models of $\mathrm{SCI}$. While rat models of SCI have been widely used, mouse models have received less attention, even though mouse models can have significant advantages over rat models. The small size of mice equates to lower animal maintenance costs than for rats, and the availability of numerous transgenic mouse models is advantageous for many types of studies. Inducing repeatable and precise injury in the animals is the primary challenge for $\mathrm{SCl}$ research, which in small rodents requires high-precision surgery. The transection-type injury model has been a commonly used injury model over the last decade for transplantation-based therapeutic research, however a standardized method for inducing a complete transection-type injury in mice does not exist. We have developed a surgical protocol for inducing a complete transection type injury in C57BL/6 mice at thoracic vertebral level 10 (T10). The procedure uses a small tip drill instead of rongeurs to precisely remove the lamina, after which a thin blade with rounded cutting edge is used to induce the spinal cord transection. This method leads to reproducible transection-type injury in small rodents with minimal collateral muscle and bone damage and therefore minimizes confounding factors, specifically where behavioral functional outcomes are analyzed.

\section{Introduction}

Spinal cord injury $(\mathrm{SCl})$ is a complex medical problem that results in drastic changes in health and lifestyle. There is no cure for $\mathrm{SCl}$, and the pathophysiology of $\mathrm{SCl}$ is not thoroughly understood. Animal $\mathrm{SCl}$ models, in particular rodent models, offer an invaluable tool for trialing new treatments, and have been used to explore $\mathrm{SCl}$ for decades. To date, over $72 \%$ of pre-clinical SCl studies have employed rat models, as compared to a mere $16 \%$ that have used mice ${ }^{1}$. Although rats, due to their larger size and tendency to form cavities akin to human SCls, have traditionally been the preferred model animals for studying novel therapeutic approaches, mice (including many transgenic mouse models) are now being used more frequently to study cellular and molecular mechanisms of the $\mathrm{SCl}^{2}$. The mouse model offers additional benefits in terms of easier handling, faster reproductive rates and lower costs than rats; mice also exhibit a high degree of genomic similarity with humans ${ }^{1,2,3}$. The major disadvantage of the mouse model has been identified as the significantly smaller size that creates challenges for surgical interventions for creating and treating spinal cord injuries ${ }^{4,5}$.

There is a gap in the existing literature that highlights the need for a robust and reproducible surgical protocol to induce stable $\mathrm{SCl}$ in the mouse model. Therefore, we provide a novel and precise surgical approach in this protocol to overcome these limitations. This protocol provides in-depth guidelines to induce a transection-type injury in mice, as this injury type has been recognized to be the most appropriate to study regenerative and degenerative changes following an injury ${ }^{6}$, as well as neuroplasticity, neural circuitry and tissue engineering approaches ${ }^{7}$. We have chosen to induce the injury in the lower thoracic region, since thoracic level $\mathrm{SCl}$ is used most commonly in the literature ${ }^{1}$.

\section{Protocol}

All procedures were carried out with the approval of the Griffith University Animal Ethics Committee (ESK/04/16 AEC and MSC/04/18 AEC) under the guidelines of the National Health and Medical Research Council of Australia.

\section{Animal set-up procedure for the surgery}

1. Anesthetize and stabilize the animal.

1. Use 8-10-week old female C57BL/6 mice. Use $5 \%$ isoflurane in $1 \mathrm{~L} / \mathrm{min}$ oxygen for induction of anesthesia. For maintenance of anesthesia, use $1.5-2 \%$ isoflurane in $1 \mathrm{~L} / \mathrm{min}$ oxygen. Confirm appropriate anaesthetization by establishing a lack of pain reflex in tail and hind paws. 
2. Keep the animal's body temperature steady at $37^{\circ} \mathrm{C}$ with a heating pad.

1. Shave the back fur to expose the surgical area over the dorsal spine. Remove the shaved fur from surgical area before sterilizing the incision site. Sterilize the shaved area with sterile cotton swabs soaked in povidone iodine antiseptic liquid and surgical spirit.

3. Administer buprenorphine $(0.03 \mathrm{mg} / \mathrm{kg}$ body weight) for analgesia and enrofloxacin ( $10 \mathrm{mg} / \mathrm{kg}$ body weight) for antibiotic cover, subcutaneously according to body weight.

4. Tape the paws of the mouse to the surgical area to stabilize the animal (Figure 1A). Place an oval window drape over mouse (Figure 1B).

\section{Laminectomy}

1. Make a vertical midline incision at the T10 vertebral level using a surgical scalpel.

1. Locate the spinous process of T10 vertebra to determine the site of laminectomy. The body of the vertebra lies slightly cranial to the tip of the spinous process ${ }^{8}$ (see Figure 2). The tip rests approximately at the midpoint of the T11 vertebra ${ }^{8}$.

2. Make the incision $\sim 2.5 \mathrm{~cm}$ long, so that the spine of T10 vertebra is approximately at the middle of the length of the incision.

2. Reflect the skin and retract with retractors.

1. Use the straight forceps to lift the skin from the underlying fascia. This will create space for the retractors to be placed; these will keep the surgical field open.

3. Perform blunt dissection of subcutaneous tissue and fascia to expose the spinous processes.

1. Use the blunt edge of the scalpel to make a small midline incision in the subcutaneous tissue and underlying fascia to expose the spines of T9-T11 vertebrae. Use the fine tip forceps (non-sharp) to perform blunt dissection and reflect the fascia.

4. Split and separate the para-spinous muscles to expose the laminae.

1. Use the blunt tip of the scalpel to split the dorsal trunk muscles and para-spinous muscles along the spines of the T9-T11 vertebrae. Use the blunt fine tip forceps to perform blunt dissection of the muscles in layers and expose the laminae of the vertebrae. This should minimize any bleeding.

NOTE: If there is any bleeding, use warmed saline $\left(37^{\circ} \mathrm{C}\right)$ irrigation and cotton swabs to control it and clear blood from the surgical field.

2. Use the same forceps to make small pockets around the transverse processes of T10 vertebra. Use the curved forceps to stabilize the T10 vertebral body by hooking its prongs under the transverse processes, in the pockets created (Figure 1C).

3. Thoroughly rinse the T10 laminae with warm saline and gently wipe clean with cotton swabs to clearly visualize the bony surface. Make sure that no muscle/ligament attachments remain along the surface bilaterally.

5. Use a drill with a fine tip ( $0.55 \mathrm{~mm}$ diameter, $7 \mathrm{~mm}$ length) to break the laminae bilaterally.

1. Use the tip of the drill to trace a vertical path from T9-T10 intervertebral space to T10-T11 intervertebral space along both the T10 laminae, without turning the drill on. This is to ensure that the drill bit does not catch any tissue (Figure 1D).

2. Now turning the drill on, slowly and carefully dig a vertical trench on the right lamina of the T10 vertebra. This part of the laminectomy should create a precise surgical defect throughout the thickness of the bone in a straight vertical line. Maintain the grip with the curved forceps to keep the vertebra stable.

6. Make sure that the tip of the drill does not penetrate through the bone and injure the spinal cord. Repeat the same process on the left side of the lamina, keeping the vertebra stable with the curved forceps. Irrigate with warm saline to wash away any remaining bone fragments.

7. Lift and remove the posterior part of the neural arch (Figure 1F).

1. Use the angled fine tip forceps to grip the spinous process and remove the whole dorsal segment of the laminae separated by the bilateral drilling. Irrigate and swab again if there is any bleeding, to clearly visualize the spinal cord exposed under the laminectomy window (Figure 1E).

\section{Transection}

1. Induce the spinal cord injury by transection of the exposed cord with a single slice of the blade.

1. Use the narrow, round cutting edged blade to slice the cord at the center of the laminectomy window. Ensure to sweep the lateral recesses of the spinal column to induce a complete transection injury (Figure 1G).

2. Confirm completeness of the transection injury using the blunt fine tip forceps and remove any remaining connections at the transection site.

3. Control the bleeding if any, before closing the surgical layers.

4. Use the warm saline to irrigate and clear any bleeding that occurs from the transected cord stumps. Use a cotton swab to apply gentle pressure to achieve hemostasis if needed. Take care not to compress the spinal cord.

\section{Closure and immediate post-operative care}

1. Bring the muscles together and suture in layer.

1. Once hemostasis is achieved at the transection site, release the curved forceps grip on the T10 vertebrae. Bring the edges of dissected muscles together along the midline to achieve good apposition.

2. Suture the muscles in a layer using 5-0 polyglactin 910 absorbable sutures. Make sure that the natural curvature of the spine does not cause any tension at the suture line or open up the sutures, exposing laminectomy site.

2. Close subcutaneous tissue and skin. 
1. Use 5-0 non-absorbable silk sutures to close the skin incision. Make sure that there is no bleeding, clots or debris remaining under the skin before closure. A final irrigation with warm saline may be necessary at this step.

3. Stop the anesthesia. Observe the animal for 10-30 min until recovery. The animal should remain on the heating pad for this duration. Provide water gel and hydrated food in the cage.

4. For post-operative care, include buprenorphine (twice daily), enrofloxacin (once daily) for the first two days prophylactically. Additionally, manually empty the bladder at least twice daily, adhering to the animal ethics committee's guidelines.

NOTE: The animals in this experiment were assessed twice daily for their general health and wellbeing, which included checking for persisting pain (to give additional doses of buprenorphine) or infection (additional enrofloxacin), their nutrition and hydration status (give injectable fluids if dehydrated) and any signs of autotomy (provide wound care if minor autotomy). It is strongly recommended that these aspects of post-operative care, including the euthanasia decisions must be determined with the guidance of the institutional animal ethics committee.

\section{Evaluation of the injury model}

1. Establishing the loss of motor function.

1. Assess motor behavior on the injured mice 2, 7, 14, 21 and 28 days after the injury in an open field using the Basso Mouse Scale (BMS) to determine the loss of function ${ }^{9}$ (Figure 3C-E).

2. Histological confirmation of injury

1. Harvest the injured spinal cords with the vertebral columns after euthanasia (done with carbon dioxide in this experiment, as approved by the animal ethics committee).

2. Fix the tissue by overnight submersion in $4 \%$ paraformaldehyde and decalcify the bones by treatment with $20 \%$ ethylene diamine tetraacetic acid (EDTA) over 3 weeks, replacing fresh EDTA every $48 \mathrm{~h}$.

3. Prepare the decalcified spines for cryo-sectioning and cut them into $30 \mu \mathrm{m}$ thick sections.

4. Mount the sections on gelatin-coated glass slides for immuno-staining with anti-GFAP antibodies and Hoechst 33342 .

5. Image the slides on a fluorescent microscope (Figure $\mathbf{3 A}$ ) and perform measurements of the injury size using image analysis software (e.g., Nikon analysis software - NIS Elements [Figure 3B]).

\section{Representative Results}

The resulting method as depicted in Figure 1, involves adequate stabilization of the mouse (Figure 1A) and good visualization of the spine and paraspinous tissue (Figure 1B). Spinous process and laminae can be clearly visualized with minimal muscle dissection and blood loss (Figure 1C, highlighted zone). The fine tip drilling is performed as shown in Figure 1D, to create a laminectomy window as shown by the rectangle. The resulting laminectomy window is clear and allows direct visualization and access to the spinal cord (Figure 1E, highlighted zone). The schematic concept of this process is explained in Figure 1F. The narrow transection blade can easily fit through the discrete laminectomy window (Figure 1G) and in a smooth swiping motion, a complete transection type injury can be created (Figure $1 \mathrm{H}-\mathrm{I}$ ). Thus, this method causes minimal muscle dissection, minimal collateral bone damage, and results in a stable complete transection type injury with minimal blood loss. Despite the induction of severe $\mathrm{SCl}$ in the animals, the surgical procedure and post-operative care resulted in high survival rates of the animals. All animals reported for this manuscript survived the spinal cord surgery; and subsequent surgeries by our lab have resulted in a survival rate of $>99 \%$.

To assess whether this method for inducing transection-type $\mathrm{SCl}$ in mice was reproducible and consistent, we analyzed the injured spinal cord using histology/immunohistochemistry and behavioral testing ( $n=8$ animals) (Figure 2). Immunolabeling against the astrocyte marker glial fibrillary acidic protein (GFAP) demarcated the boundary of the intact spinal cord, with the injury site being the region between the cord stumps when viewed with longitudinal sections (Figure 3A). A consistent-sized defect was induced at the transection site, with an average minimal distance of $550.4 \pm 17.3 \mu \mathrm{m}$ (Figure 3B). Behavioral data deploying the Basso Mouse Scale (BMS) ${ }^{9}$ of an open field test showed that the injured mice exhibit no hind limb movement after the injury (Figure 3C). This is represented by a score of 0 on the BMS for up to 28 days post-injury. Thus, the protocol produces complete and reliable transection-type injury resulting in complete loss of function below the injury level and does not lead to spontaneous reversal of the paralysis (Figure 3D,E). 

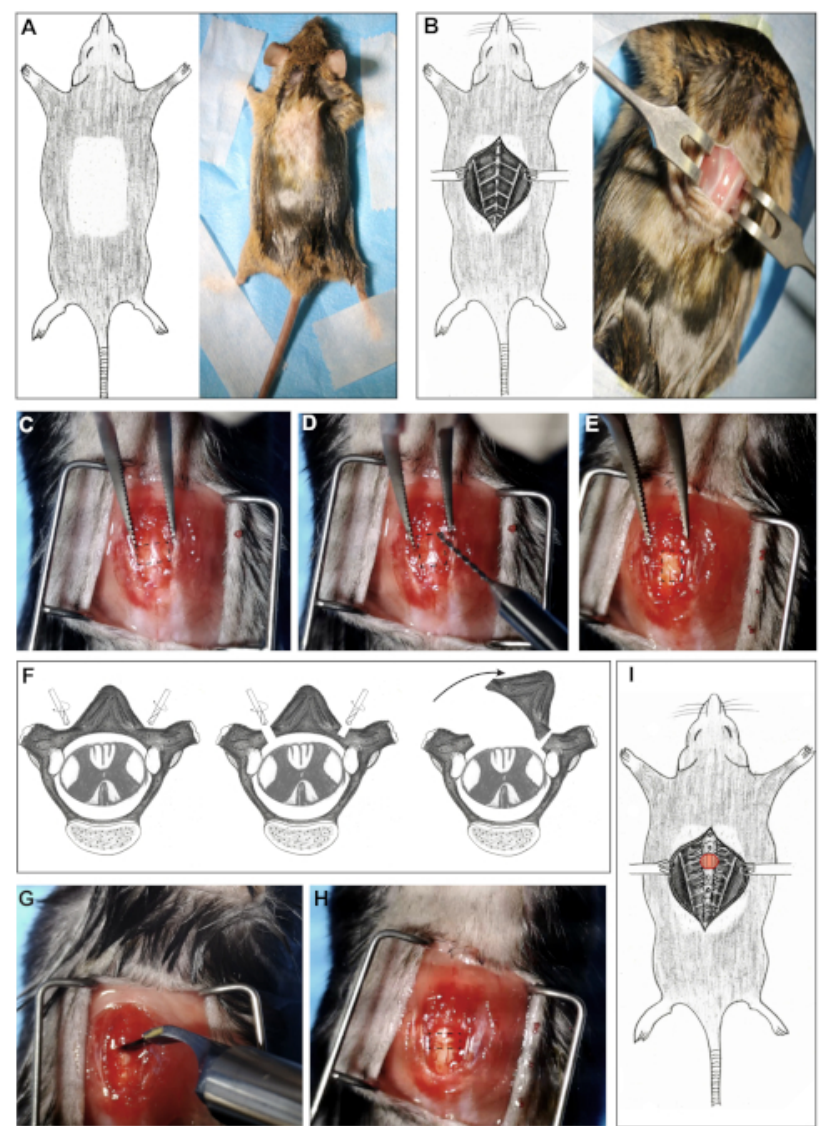

Figure 1: Key steps in the transection injury protocol. (A) Animal set up and stabilization prior to surgery. Schematic and photograph from the surgery are both shown. (B) Schematic and photograph showing incision, retractor placement and blunt dissection to expose the deep muscle layers. (C) Stabilization of spine with forceps and exposing laminae of T10 vertebra. The rectangle in the photograph highlights laminae and spinous process of the T10 vertebra. (D) Fine tip drill to perform laminectomy. The rectangle in this photograph traces the laminectomy window. (E) Complete laminectomy window highlighted by the rectangle, within which the spinal cord is clearly visible. (F) Series of schematic drawings showing the drilling concept to perform a complete laminectomy, in a cross-sectional orientation. (G) This photograph depicts the use of a fine blade transection knife to perform the complete transection type injury, and in $(\mathbf{H})$ the complete injury is visible inside the rectangle, as a dark red transverse line across the spinal cord. (I) Schematic showing the overall view of the laminectomy and injury site. Please click here to view a larger version of this figure.

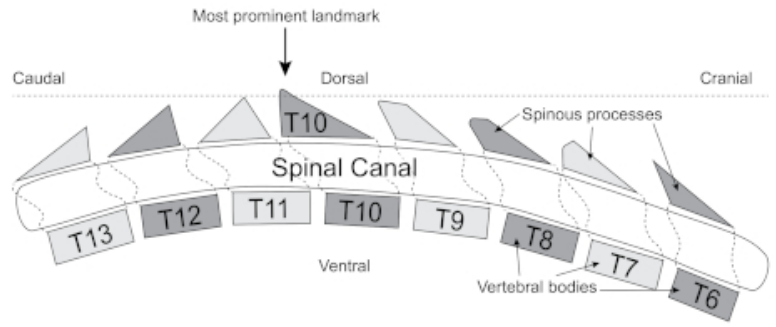

Figure 2: A schematic explaining the vertebral landmark identification for T10. The spinous process of T10 vertebra is one of the most prominent landmarks that can be palpated at the natural curvature of the thoracic spine. At this point the spinous processes change the morphology so that the tips of the spinous processes cranial from T10 point caudally, and the tips of the spinous processes caudal from T10 point cranially. Please click here to view a larger version of this figure. 

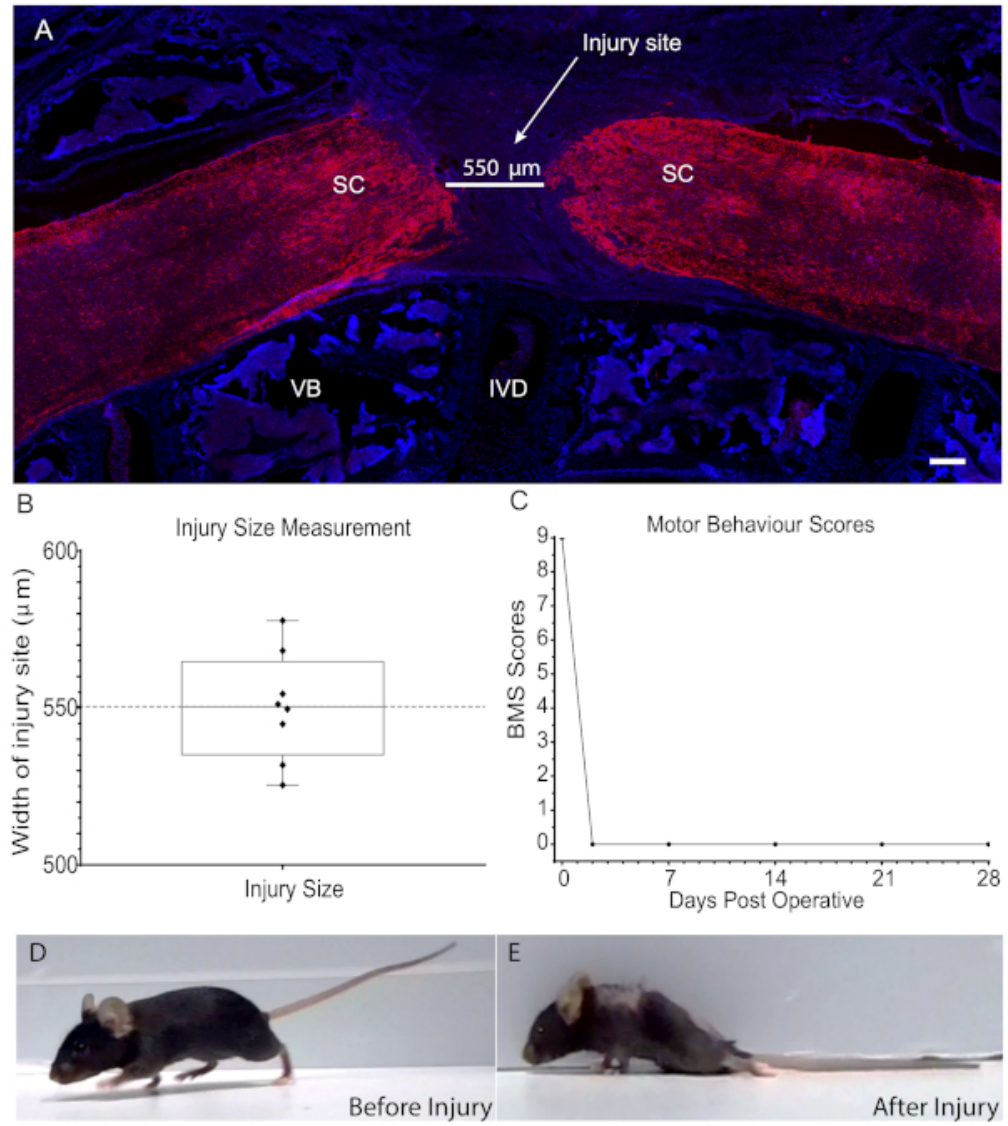

Figure 3: Representative results from transection type injury induced in C57BL/6 mice. (A) A longitudinal section of the spinal cord reveals the complete transection-type injury. Tissue was imaged on an inverted microscope. Anti-GFAP immunolabelling labels astrocytes (red) while nuclei are labeled with Hoechst 33342 (blue). The injury gap using a linear measurement of the shortest point was $550 \mu \mathrm{m}$. Scale bar $=200 \mu \mathrm{m}$. IS = injury site, IVD = intervertebral disc, SC = spinal cord, VB = vertebral body. (B) Injury size was measured in 8 animals. The mean injury size was $550.4 \pm 17.3 \mu \mathrm{m}$ with maximum being $577.8 \mu \mathrm{m}$ and minimum $525.4 \mu \mathrm{m}$. (C) Motor behavior scored on Basso Mouse Scale (BMS), which was 9 in all mice prior to the injury and remained at 0 for 4 weeks after the injury, indicating a complete loss of motor function below the injury site $(n=8$ mice). (D) Gait of a healthy mouse before the injury. (E) Gait of the same mouse after the injury. Please click here to view a larger version of this figure.

\section{Discussion}

This method induces a complete transection type injury at the T10 vertebral level in mice, which results in complete paraplegia of the animal, below the level of injury. Overall, this method results in minimal bleeding, negligible collateral damage and a stable, reproducible injury. As compared to previously published methods of transection without laminectomy ${ }^{10}$, this method offers the benefits in terms of direct visualization without manipulating the curvature of the spine, better control over completeness of the injury, and enhanced ability to control bleeding and achieve hemostasis. The advantage of this method is that the protocol can be modified for use at any other vertebral levels other than T10, as well as to perform other injury types such as hemi-sections, partial dorsal transections, dorsal root avulsions, crush and contusions.

A major component of this protocol is that it employs the use of a fine-tip drill. While the use of the drill may require a high skill level and more extensive training, it achieves a clean and complete laminectomy. Another crucial factor is the use of a narrow-bladed knife for transection, instead of micro-scissors. This results in less unwanted collateral damage as compared to using scissors. Conversely, however, if too much lateral pressure is exerted, the blade can cause some injury to the vertebral body. The described protocol may require the surgeon to perform some troubleshooting. If the laminectomy is not performed properly, there may be bone spurs remaining, which can restrict access to the laminectomy window. Inserting one of the prongs of the fine tip forceps can enable the surgeon to grasp and break off any remaining bone spurs. However, care must be taken to not injure the exposed spinal cord in the process. If the laminectomy results in a jagged bone edge, the drilling can be performed again to straighten the laminectomy margin. This may be impractical if the laminectomy window is already wide enough, in which case, the transection should be performed without tampering with the laminectomy site.

It is strongly recommended that the users practice the laminectomy procedures at least 8-10 times at the relevant spinal level in a cadaveric dissection prior to attempting in a live survival surgery. Although, the drill holding and maneuvering techniques are simple, they may require the users to get acquainted with the equipment. Here, we also provide some useful advice to help with spine and hand stabilization during the drilling procedure. If the user is right handed, the spine should be stabilized by using the curved forceps with the left hand so that the forceps approach the spine from the cranial aspect. This keeps the caudal aspect of the spine clear to approach with the drill held in the right (or the predominant) hand. The drill should be held with a pincer grip between thumb, index and middle fingers. The hand should be well supported along the medial 
edge of the wrist and the outstretched fifth finger. Keeping the arm completely adducted so that the elbow touches the body may help achieve better control over the drill grip during practice. The drilling action should only involve motion at the fingers holding the drill and not at the wrist, not unlike using a pen for writing.

\section{Disclosures}

The authors have nothing to disclose.

\section{Acknowledgments}

This work was supported by a Griffith University International Student (PhD) stipend to RR, a Perry Cross Foundation Grant to JE and JSJ, a Clem Jones Foundation Grant to JSJ and JE, and a Motor Accident Insurance Commission of Queensland grant to JSJ and JE.

\section{References}

1. Sharif-Alhoseini, M. et al. Animal models of spinal cord injury: a systematic review. Spinal Cord. 55 (8), 714-721, (2017)

2. Lee, D. H., Lee, J. K. Animal models of axon regeneration after spinal cord injury. Neuroscience Bulletin. 29 (4), 436-444, (2013).

3. Sharif-Alhoseini, M., Rahimi-Movaghar, V. in Topics in Paraplegia. 10.5772/57189 (ed Yannis Dionyssiotis) IntechOpen, July 2nd (2014).

4. Talac, R. et al. Animal models of spinal cord injury for evaluation of tissue engineering treatment strategies. Biomaterials. 25 (9), 1505-1510, (2004).

5. Nakae, A. et al. The animal model of spinal cord injury as an experimental pain model. Journal of Biomedicine \& Biotechnology. 2011 939023, (2011).

6. Kwon, B., Oxland, T., Tetzlaff, W. Animal models used in spinal cord regeneration research. Spine. 27 1504-1510, (2002).

7. Kundi, S., Bicknell, R., Ahmed, Z. Spinal cord injury: current mammalian models. American Journal of Neuroscience. (4), 1-12, (2013).

8. Harrison, M. et al. Vertebral landmarks for the identification of spinal cord segments in the mouse. Neuroimage. 68 22-29, (2013).

9. Basso, D. M. et al. Basso Mouse Scale for locomotion detects differences in recovery after spinal cord injury in five common mouse strains. Journal of Neurotrauma. 23 (5), 635-659, (2006).

10. Seitz, A., Aglow, E., Heber-Katz, E. Recovery from spinal cord injury: a new transection model in the C57BI/6 mouse. Journal of Neuroscience Research. 67 (3), 337-345, (2002). 